\title{
Syphilis Infection during Pregnancy: Fetal Risks and Clinical Management
}

\author{
Marco De Santis, ${ }^{1}$ Carmen De Luca, ${ }^{1}$ Ilenia Mappa, ${ }^{1}$ Terryann Spagnuolo, ${ }^{1}$ Angelo Licameli, ${ }^{1}$ \\ Gianluca Straface, ${ }^{2}$ and Giovanni Scambia ${ }^{1}$ \\ ${ }^{1}$ Department of Obstetrics and Gynaecology, Università Cattolica del Sacro Cuore, Policlinico "A. Gemelli", Rome, Italy \\ ${ }^{2}$ Department of Obstetrics and Gynaecology, Policlinico Abano Terme, Abano Terme (PD), Italy \\ Correspondence should be addressed to Marco De Santis, marcodesantis@rm.unicatt.it
}

Received 15 March 2012; Accepted 17 May 2012

Academic Editor: Francesco De Seta

Copyright (C) 2012 Marco De Santis et al. This is an open access article distributed under the Creative Commons Attribution License, which permits unrestricted use, distribution, and reproduction in any medium, provided the original work is properly cited.

\begin{abstract}
Congenital syphilis is still a cause of perinatal morbidity and mortality. Untreated maternal infection leads to adverse pregnancy outcomes, including early fetal loss, stillbirth, prematurity, low birth weight, neonatal and infant death, and congenital disease among newborns. Clinical manifestations of congenital syphilis are influenced by gestational age, stage of maternal syphilis, maternal treatment, and immunological response of the fetus. It has been traditionally classified in early congenital syphilis and late congenital syphilis. Diagnosis of maternal infection is based on clinical findings, serological tests, and direct identification of treponemes in clinical specimens. Adequate treatment of maternal infection is effective for preventing maternal transmission to the fetus and for treating fetal infection. Prenatal diagnosis of congenital syphilis includes noninvasive and invasive diagnosis. Serological screening during pregnancy and during preconception period should be performed to reduce the incidence of congenital syphilis.
\end{abstract}

\section{Introduction}

Syphilis is a sexually transmitted disease (STD) caused by the bacterium Treponema pallidum, but little is known about its mechanism of action or what determines virulence of infection [1]. Untreated syphilis in pregnancy leads to adverse outcomes among more than half of the women with active disease, including early fetal loss, stillbirth, prematurity, low birth weight, neonatal and infant death, and congenital disease among newborn babies [2]. In 2010, a total of 13,774 cases of primary and secondary syphilis were reported to Centers for Disease Control and Prevention [3]. According to World Health Organization (WHO), 12 million people were infected each year [4]. It was estimated that the lifetime medical cost per case of syphilis is \$572 (in year 2006 dollars) and they could be much higher if CS and HIV infections occurred [5]. Screening and early detection can reduce these costs because treatment for early stage syphilis is less expensive than treatment for later stage disease: $\$ 41.26$ (in year 2001 dollars) compared to $\$ 2,061.70$ for late syphilis [6].
Moreover, CDC recommends that all persons who have syphilis should be tested for HIV infection [7]. Genital sores caused by syphilis can bleed easily and make it easier to transmit HIV infection, with a 2- to 5-fold increased risk of acquiring HIV [8]. Changes in the population incidence of primary and secondary syphilis among women are usually followed by similar changes in the incidence of congenital syphilis (CS) [9]. CDC reported that the rates of both female and CS increased during 2005-2008 in the United States of America (USA), and have since declined. The rate of syphilis among women was 1.1 cases per 100,000 women in 2010, and the rate of CS was 8.7 cases per 100,000 live births in 2010 [10]. According to the most recent (2008) estimates from WHO, about 1.9 million pregnant women had active syphilis [11]. In Italy, the incidence rate of syphilis was 0.86 per 100,000 population in 2008 [12], and CS is strictly related to immigration, mostly from Eastern Europe. In 2007 an Italian prospective study on 19.548 pregnant women showed that the overall syphilis seroprevalence was $0.44 \%$ but it was 4.3\% in women from Eastern Europe and 5.8\% in women 
from Central-South America. This high prevalence could be explained by the fact that immigrant women do not always receive adequate prenatal care owing to lack of knowledge of free healthcare services [13].

Without screening and treatment, about $70 \%$ of infected women will have an adverse pregnancy outcome [14].

\section{Clinical Manifestations}

Clinical manifestations of acquired syphilis are not apparently altered by pregnancy. Syphilis is passed from person to person through direct contact with a syphilitic sore, called chancre. Transmission of the organism occurs during vaginal, anal, or oral sex. Sores of primary syphilis occur about 3 weeks after contact, mainly on the external genitals, vagina, cervix, anus, or in the rectum. They are often unrecognized in women because they can be asymptomatic. Syphilitic sore is firm, round, small, and painless and lasts 3 to 6 weeks. It should be distinguished by Genital Herpes, which causes small, painful blisters filled with clear or straw-colored fluid. When blisters break, they leave shallow ulcers that are very painful and eventually crust over and slowly heal over 7-14 days or more [2].

Sypilitic sore can increase the risk of HIV transmission by disrupting mucosal and epithelial barriers [15] and is followed several weeks or months later by widespread cutaneous, mucosal, and sometimes systemic indications of the dissemination of the spirochetes of secondary syphilis. This phase can last up to a year and syphilis is particularly contagious at this stage. Even without treatment both primary and secondary lesions resolve and the infection enters a latent stage. Despite the lack of clinical manifestations, the infection can still be transmitted to the fetus [2]. Tertiary syphilis may occur in a third of untreated people, approximately three to 15 years after the initial infection. It is characterized by infiltrative tumors of skin, bones, or liver (gumma) (15\%), central nervous system disorders (neurosyphilis) (6.5\%), and cardiovascular problems (10\%). People with tertiary syphilis are not infectious [16].

\section{Fetal Infection}

Spirochetes can cross the placenta and infect the fetus from about 14 weeks' gestation, and the risk of fetal infection increases with gestational age [17]. The manifestations of CS are influenced by gestational age, stage of maternal syphilis, maternal treatment, and immunological response of the fetus [18]. CS can lead to spontaneous abortion, usually after the first trimester, or late-term stillbirth in 30 to 40 percent of cases or premature or term delivery of live infants who may have obvious signs of infection or be fully asymptomatic (approximately two-thirds of liveborn cases) [19]. Placental infection and the reduction in blood flow to the fetus are the most common causes of fetal death. An untreated woman has about $70 \%$ of chance of fetal infection during the first 4 years of disease. In $35 \%$ of cases, infected fetuses are born alive with CS. Low birth weight can be the only sign of infection. In fact about $60 \%$ of liveborns are asymptomatic at birth [20, 21]. CS has been traditionally classified in early congenital syphilis (ECS) and late congenital syphilis (LCS). In ECS signs appear in the first 2 years of life while in LCS signs appear over the first 2 decades. Clinical manifestations of ECS are the result of active infection and inflammation while clinical manifestations of LCS are malformation or stigmata that represent the scars induced by initial lesions of ECS or can be the result of chronic inflammation [2]. After fetal infection occurs, any organ system can be affected because of the widespread spirochetal dissemination.

\section{Early Congenital Syphilis}

Hepatomegaly is present in nearly all infants with CS, while splenomegaly is present in half of cases. Jaundice has been recorded in $33 \%$ of cases, as a consequence of syphilitic hepatitis or of hemolytic anemia $[2,22]$. Elevated serum transaminase and alkaline phosphatase concentrations and direct hyperbilirubinemia can occur; the prothrombin time may be prolonged [23-25]. Generalized lymphadenopathy has been described in 50\% of patients. Large epitrochlear nodes are typical of CS [22].

Hematological manifestations, such as anemia, thrombocytopenia, leukopenia, and leukocytosis are common findings in CS [2]. Hydrops fetalis may also be a manifestation. In presence of a negative coomb's test in a hydropic infant with hemolytic anemia CS should be considered [26].

Mucocutaneous involvement occurs in as many as $70 \%$ of infected infants and may be present at birth or develop during the first few weeks of life. The most common cutaneous manifestation consists of small copper-red maculopapular lesions, and the hands and feet often are most severely affected. Desquamation and crusting occur over the course of 1 to 3 weeks $[2,22]$. Rhinitis may be an early symptom which appears after the first week of life and usually before the end of the third month. Mucus discharge is often is blood-tinged and secondary bacterial infections can occur. "Saddle nose" deformity is one of the later stigmata of the disease, and can occur when ulceration of nasal mucosa involves the nasal cartilage. All mucocutaneous lesions and discharges contain abundant spirochetes and are highly infectious. After the first 2 or 3 months of age, the perioral and the perineal area may be affected by wart-like or flat lesions called condyloma lata that can lead to deep fissures and can result in fine scars called rhagades $[2,27]$. Petechial lesions may be seen if severe thrombocytopenia is present [22].

Bone involvement is very frequent in untreated ECS. The metaphyseal and diaphyseal portions of long bones are usually affected by periostitis and cortical demineralization, while osteochondritis involves the joints, especially knees, ankles, wrists, and elbows. Osteochondritis and periostitis may be painful and manifested by the pseudoparalysis of a limb due to pain (pseudoparalysis of Parrot), which affects more frequently the upper extremities [28].

A nephrotic syndrome can appear at 2 or 3 months of age, and it can lead to generalized edema [2].

Congenital neurosyphilis may be asymptomatic. More than $25 \mathrm{WBC} / \mathrm{mm}^{3}$ and protein greater than $150 \mathrm{mg} / \mathrm{dL}$ $(170 \mathrm{mg} / \mathrm{dL}$ in premature infants) in cerebrospinal fluid (CSF) are considered suggestive of neurosyphilis although 
normal CSF indices do not exclude neurosyphilis. A reactive CSF Venereal Disease Research Laboratory (VDRL) test generally indicates the presence of neurosyphilis. CSF abnormalities are present in approximately 8 percent of asymptomatic infants born to mothers with untreated early syphilis [2, 29, 30].

Ocular manifestations are rare and include chorioretinitis, glaucoma, uveitis, cataract, salt and pepper fundus, and chancres of the eyelid. Other findings are less common [2].

\section{Late Congenital Syphilis}

LCS is actually very rare and occurs in approximately 40 percent of untreated children $[2,30]$.

Syphilitic vasculitis around the time of birth can lead to dental abnormalities that occur in teeth that undergo calcification during the first year of life. Hutchinson's teeth are peg-shaped, notched central incisors while mulberry molars are multicuspid first molars. The deciduous teeth have an increased risk of dental caries [30, 31].

Interstitial keratitis is the typical ocular manifestation, usually diagnosed between 5 and 20 years of age. It can lead to secondary glaucoma or corneal clouding [2].

Eight nerve deafness occurs in 3\% of cases and is secondary to luetic involvement of the temporal bone. Eight nerve involvement can be unilateral or bilateral, and it may be responsive to corticosteroids. Although it is usually diagnosed between 30 and 40 years of age, it often occurs in the first decades [32].

The constellation of Hutchinson's teeth, interstitial keratitis and eight nerve deafness is called Hutchinson's triad [2], described by Sir Jonathan Hutchinson (1828-1913) from England. Fortunately, it actually represents a rare finding.

Syphilis rhinitis can impair the maxilla growth, resulting in an abnormal configuration in the middle section of the face, while nasal cartilage destruction resulting from inflammation can cause the perforation of the nasal septum and lead to saddle nose $[2,33]$.

Rhagades around the body orifices can result from the fissuration of early linear scares [31].

Neurological manifestations of LCS include mental retardation, hydrocephalus, convulsive disorders, cranial nerve abnormalities (including blindness and deafness), and juvenile general paresis [2, 27].

Bony involvement is less frequent than in ECS and it includes the sequelae of prolonged periostitis of the skull (resulting in frontal bossing), of the tibia (resulting in saber shin) and of the sternoclavicular portion of the clavicle (resulting in a deformity called Higouménakis sign).

Clutton's joints are symmetric, painless, sterile, synovitis usually localized to the knees and characterized by local tenderness and limitation of motion [2, 27, 31].

\section{Diagnosis}

Maternal syphilis can be suspected based on clinical findings and confirmed by direct identification of treponemes in clinical specimens and by positive serologic findings or can be accidently diagnosed through screening serological tests.
Dark field microscopy is the most specific technique for diagnosing syphilis when an active chancre or condyloma latum is present [34]. Other possible methods include direct fluorescent antibody (DFA) testing and the rabbit infectivity test (not used in clinical practice) [2]. Serological tests for syphilis can be classified in nontreponemal (NTTs) and treponemal (TTs) tests. NTTs are usually used for screening and monitoring therapy, while TTs are used to confirm the diagnosis. Nontreponemal tests detect antibodies to cardiolipin, a component of membranes and mammalian tissue. The two commonly used nontreponemal tests are the Venereal Disease Research Laboratory (VDRL) and the Rapid Plasma Reagin (RPR) tests. False-positive reactions can occur because of pregnancy, autoimmune disorders, and infections [35]. NTTs are usually positive in $75 \%$ of cases of primary syphilis. Secondary syphilis is always characterized by a reactive VDRL, with a titer greater than 1/16 [2]. The titer of antibodies reflects disease activity: fourfold decrease suggests adequate therapy, while fourfold increase indicates active disease. NTTs usually become negative one year after receiving adequate treatment of primary syphilis and within two years with secondary syphilis. In a small percentage of patients low positive titers persist despite receiving adequate therapy [30]. TTs detect an interaction between serum immunoglobulins and surface antigens of Treponema pallidum. They include the fluorescent treponemal antibody absorption (FTA-ABS) test, the treponemal-specific microhemagglutination test (MHATP) and Treponema pallidum particle agglutination test (TP-PA). These tests are positive in 75\% (TP-PA) to $85 \%$ (FTA-ABS) of patients with primary syphilis and in $100 \%$ of patients with secondary syphilis. False-positive tests can occur in patients with Lyme disease, leptospirosis, and diseases caused by other pathogenic Treponema spp. [2]. TTs usually remain positive for life. Polymerase-chain-reaction(PCR-) based tests and immunoglobulin $\mathrm{M}$ immunoblotting tests have been developed, but they are not largely used in clinical practice. Although no Treponema pallidum detection tests are commercially available, some laboratories provide locally developed PCR tests for the detection of Treponema pallidum [2].

Prenatal diagnosis of CS includes noninvasive and invasive diagnosis. Ultrasonographic fetal examination for signs of CS is recommended prior to therapy after 20 weeks' gestation. Fetal syphilis is the presumed diagnosis when the sonographic findings of fetal hydrops, abnormally large abdomen (hepatosplenomegaly), hydramnios, and thick placenta are found in the presence of maternal syphilis [36-38]. Invasive diagnosis includes amniocentesis and percutaneous umbilical blood sampling. Dark field examination, rabbit infectivity testing, and polymerase chain reaction for detection of Treponema pallidum can be performed on amniotic fluid. Hematologic and chemical testing can be performed on fetal blood and fetal antitreponemal IgM can be detected. Abnormal liver transaminases, anemia, and thrombocytopenia are signs of fetal infection. If fetal infection is suspected, antepartum fetal heart rate testing is indicated before treatment. In some cases of fetal hydrops, fetuses can have late decelerations or nonreactive nonstress testing that led to fetal distress soon after maternal treatment [36]. Evaluation of infants for 
suspected CS should include careful physical examination, nontreponemal serologic tests of infant serum, specimens for testing for the presence of spirochetes from mucocutaneous lesions (if these are present), complete blood count, CSF analysis (in all infants with physical findings compatible with CS quantitative nontreponemal titer $>4$-fold higher than the current maternal titer, or direct evidence of Treponema pallidum in clinical specimens), long bone radiographs (unless the diagnosis has been confirmed otherwise), adequate clinical tests in case of specific signs or symptoms, and pathologic examination of the placenta or umbilical cord [39].

\section{Treatment}

Adequate treatment of maternal infection is effective for preventing maternal transmission to the fetus and for treating fetal infection [40]. Penicillin G, administered parenterally, is the preferred drug for treating of syphilis. The effectiveness of penicillin was established through clinical experience and randomized controlled clinical trials. It provides weeks of treponemicidal levels of penicillin in the blood, but it does not efficiently cross the blood brain barrier. Aqueous crystalline penicillin $\mathrm{G}$ is the drug of choice for neurosyphilis treatment [7]. Treatment failure was described in few case reports, particularly in patients with HIV infection, but there is no documented penicillin resistance in T. pallidum [41]. CDC recommends that pregnant women should be treated with the penicillin regimen appropriate for their stage of infection [7]. Evidence is insufficient to determine optimal, recommended penicillin regimens [42]. In primary, secondary, and early latent syphilis, benzathine penicillin $G$ 2.4 million units IM in a single dose is recommended [7]. Additional therapy can be beneficial for pregnant women in some settings. Some authors suggest that a second dose of benzathine penicillin 2.4 million units IM administered 1 week after the initial dose for women who have primary, secondary, or early latent syphilis [36]. In late latent syphilis or latent syphilis of unknown duration, benzathine penicillin G 7.2 million units total should be administered, as 3 doses of 2.4 million units IM each at 1 week intervals. In case of neurosyphilis, aqueous crystalline penicillin G 18-24 million units per day, administered as 3-4 million units IV every 4 hours or continuous infusion, for 10-14 days represents the suggested treatment [7]. Pregnant women who have a history of penicillin allergy should be desensitized and treated with penicillin $[7,36]$. In case of HIV positive patients, placental inflammation from congenital infection might increase the risk for perinatal transmission of the virus. No sufficient data are available to recommend a specific regimen for HIVinfected pregnant women [7].

The Jarisch-Herxheimer reaction can occur in some patients 2 to 12 hours after receiving therapy for active syphilis. It is characterized by fever, headache, myalgia, and malaise, and it is caused by the release of treponemal endotoxin-like compounds during penicillin-mediated lysis [2, 43, 44]. The Jarisch-Herxheimer reaction can increase the risk of premature labor and/or fetal distress during the second half of pregnancy $[45,46]$. Serologic titers should be repeated at 28-32 weeks' gestation and at delivery and should be checked monthly in women at high risk for reinfection or in high-risk geographic areas [7]. Maternal treatment can be inadequate if delivery occurs within 30 days of therapy, or if the maternal antibody titer at delivery is fourfold higher than the pretreatment titer [36].

\section{Conclusions}

Syphilis infection during pregnancy still represents a worldwide public health problem. The American College of Obstetricians and Gynecologists and the American Academy of Pediatrics recommend prenatal syphilis screening at the first prenatal visit and again at 32-36 weeks, if the woman is at risk for syphilis [47]. CDC recommends that all women should be screened serologically for syphilis at the first prenatal visit and, for patients at high risk, during the third trimester and at delivery [7]. Moreover, any woman who delivers a stillborn infant after 20 weeks' gestation should be tested for syphilis [36]. The Italian Guidelines of Istituto Superiore di Santità for Physiological Pregnancy (2011) stated that serological screening for syphilis should be offered to all pregnant women during the first and the third trimester of pregnancy [48]. Preconception serological tests for syphilis could represent the key to reduce the incidence of CS. Moreover, preconception counseling could play an important role, evaluating the woman and her partner for exposure to sexually transmitted diseases, identifying high-risk behaviors, and providing health promotion messages and education.

\section{References}

[1] S. M. Berman, "Maternal syphilis: pathophysiology and treatment," Bulletin of the World Health Organization, vol. 82, no. 6, pp. 433-438, 2004.

[2] D. Ingall and P. J. Sánchez, "Syphilis," in Infectious Diseases of the Fetus and Newborn Infant, J. S. Remington and J. O. Klein, Eds., pp. 643-681, W.B. Saunders, Philadelphia, 5th edition, 2001.

[3] Centers for Disease Control and Prevention National Overview of Sexually Transmitted Diseases (STDs), http://www .cdc.gov/std/stats10/natoverview.htm, 2010.

[4] World Health Organization, The global elimination of congenital syphilis: rationale and strategy for action, http:// whqlibdoc.who.int/publications/2007/9789241595858_eng .pdf, 2007.

[5] H. W. Chesson, D. Collins, and K. Koski, "Formulas for estimating the costs averted by sexually transmitted infection (STI) prevention programs in the United States," Cost Effectiveness and Resource Allocation, vol. 6, no. 1, article 10, 2008.

[6] J. M. Blandford and T. L. Gift, "The cost-effectiveness of single-dose azithromycin for treatment of incubating syphilis," Sexually Transmitted Diseases, vol. 30, no. 6, pp. 502-508, 2003.

[7] K. A. Workowski and S. Berman, "Sexually transmitted diseases treatment guidelines," Morbidity and Mortality Weekly Report, vol. 59, no. RR-12, pp. 1-113, 2010.

[8] Centers for Disease Control and Prevention, Syphilis-CDC Fact Sheet, http://www.cdc.gov/std/syphilis/STDFact-Syphilis .html, 2012.

[9] Centers for Disease Control and Prevention, "Congenital syphilis-United States, 2003-2008," Morbidity and Mortality Weekly Report, vol. 59, no. 14, pp. 413-417, 2010. 
[10] Centers for Disease Control and Prevention, Sexually Transmitted Diseases Surveillance, http://www.cdc.gov/std/stats10/ Syphilis.htm, 2010.

[11] World Health Organization, "Towards eliminating congenital syphilis," Progress Report, 2011, http://www.who.int/reproductivehealth/topics/rtis/GlobalData_cs_pregnancy2011.pdf.

[12] World Health Organization, Regional Office for Europe. Sexually transmitted infections, http://data.euro.who.int/cisid/ ?TabID=284849, 2012.

[13] E. Tridapalli, M. G. Capretti, V. Sambri et al., "Prenatal syphilis infection is a possible cause of preterm delivery among immigrant women from eastern Europe," Sexually Transmitted Infections, vol. 83, no. 2, pp. 102-105, 2007.

[14] S. Hawkes, N. Matin, N. Broutet, and N. Low, "Effectiveness of interventions to improve screening for syphilis in pregnancy: a systematic review and meta-analysis," The Lancet Infectious Diseases, vol. 11, no. 9, pp. 684-691, 2011.

[15] R. M. Greenblatt, S. A. Lukehart, F. A. Plummer et al., "Genital ulceration as a risk factor for human immunodeficiency virus infection," AIDS, vol. 2, no. 1, pp. 47-50, 1988.

[16] M. E. Kent and F. Romanelli, "Reexamining syphilis: an update on epidemiology, clinical manifestations, and management," Annals of Pharmacotherapy, vol. 42, no. 2, pp. 226-236, 2008.

[17] R. L. Goldenberg and C. Thompson, "The infectious origins of stillbirth," American Journal of Obstetrics and Gynecology, vol. 189, no. 3, pp. 861-873, 2003.

[18] H. Saloojee, S. Velaphi, Y. Goga, N. Afadapa, R. Steen, and O. Lincetto, "The prevention and management of congenital syphilis: an overview and recommendations," Bulletin of the World Health Organization, vol. 82, no. 6, pp. 424-430, 2004.

[19] H. B. Jensen, "Congenital syphilis," Seminars in Pediatric Infectious Diseases, vol. 10, pp. 183-194, 1999.

[20] D. Watson-Jones, J. Changalucha, B. Gumodoka et al., "Syphilis in pregnancy in Tanzania. I. Impact of maternal syphilis on outcome of pregnancy," Journal of Infectious Diseases, vol. 186, no. 7, pp. 940-947, 2002.

[21] J. S. Sheffield, P. J. Sánchez, G. Morris et al., "Congenital syphilis after maternal treatment for syphilis during pregnancy," American Journal of Obstetrics and Gynecology, vol. 186, no. 3, pp. 569-573, 2002.

[22] F. Saxoni, P. Lapaanis, and S. N. Pantelakis, "Congenital syphilis: a description of 18 cases and re-examination of an old but ever-present disease," Clinical Pediatrics, vol. 6, no. 12, pp. 687-691, 1967.

[23] D. H. Dorfman and J. H. Glaser, "Congenital syphilis presenting in infants after the newborn period," The New England Journal of Medicine, vol. 323, pp. 1299-1302, 1990.

[24] M. C. Shah and L. L. Barton, "Congenital syphilitic hepatitis," Pediatric Infectious Disease Journal, vol. 8, no. 12, pp. 891-892, 1989.

[25] W. A. Long, M. H. Ulshen, and E. E. Lawson, "Clinical manifestations of congenital syphilitic hepatitis: implications for pathogenesis," Journal of Pediatric Gastroenterology and Nutrition, vol. 3, no. 4, pp. 551-555, 1984.

[26] S. I. Bulova, E. Schwartz, and W. V. Harrer, "Hydrops fetalis and congenital syphilis," Pediatrics, vol. 49, no. 2, pp. 285-287, 1972.

[27] R. V. Platou, "Treatment of congenital syphilis with penicillin," Advances in Pediatrics, vol. 4, article 35, 1949.

[28] R. H. Wilkinson and R. M. Heller, "Congenital syphilis: resurgence of an old problem,” Pediatrics, vol. 47, no. 1, pp. 27-30, 1971.

[29] I. C. Michelow, G. D. Wendel, M. V. Norgard et al., "Central nervous system infection in congenital syphilis," The New
England Journal of Medicine, vol. 346, no. 23, pp. 1792-1798, 2002.

[30] C. R. Woods, "Syphilis in children: congenital and acquired," Seminars in Pediatric Infectious Diseases, vol. 16, no. 4, pp. 245257, 2005.

[31] D. Nabarro, Congenital Syphilis, E. Arnold, London, UK, 1954.

[32] R. Rothenberg, G. Becker, and R. Wiet, "Syphilitic hearing loss," Southern Medical Journal, vol. 72, no. 2, pp. 118-120, 1979.

[33] P. J. Sánchez and L. T. Gutman, "Syphilis," in Textbook of Pediatric Infectious Diseases, R. D. Feigin, J. D. Cherry, and G. J. Demmler, Eds., pp. 1724-1743, W.B. Saunders, Philadelphia, Pa, USA, 5th edition, 2004.

[34] S. A. Larsen, B. M. Steiner, and A. H. Rudolph, "Laboratory diagnosis and interpretation of tests for syphilis," Clinical Microbiology Reviews, vol. 8, no. 1, pp. 1-21, 1995.

[35] F. T. Fischbach, "Syphilis detection tests," in A Manual of Laboratory \& Diagnostic Tests, pp. 581-583, Lippincott, Philadelphia, 6th edition, 2000.

[36] G. D. Wendel Jr., J. S. Sheffield, L. M. Hollier, J. B. Hill, P. S. Ramsey, and P. J. Sánchez, "Treatment of syphilis in pregnancy and prevention of congenital syphilis," Clinical Infectious Diseases, vol. 35, supplement 2, pp. S200-S209, 2002.

[37] L. M. Hill and J. B. Maloney, "An unusual constellation of sonographic findings associated with congenital syphilis," Obstetrics and Gynecology, vol. 78, no. 5, pp. 895-897, 1991.

[38] L. Nathan, D. M. Twickler, M. T. Peters, P. J. Sanchez, and G. D. Wendel Jr., "Fetal syphylis: correlation of sonographic findings and rabbit infectivity testing of amniotic fluid," Journal of Ultrasound in Medicine, vol. 12, no. 2, pp. 97-101, 1993.

[39] Committee on Infectious Diseases and American Academy of Pediatrics, "Syphilis," in Red Book, pp. 595-607, American Academy of Pediatrics, Elk Grove Village, Ill, USA, 2003.

[40] J. M. Alexander, J. S. Sheffield, P. J. Sanchez, J. Mayfield, and G. D. Wendel Jr., "Efficacy of treatment for syphilis in pregnancy," Obstetrics and Gynecology, vol. 93, no. 1, pp. 5-8, 1999.

[41] L. V. Stamm, "Global challenge of antibiotic-resistant Treponema pallidum," Antimicrobial Agents and Chemotherapy, vol. 54, no. 2, pp. 583-589, 2010.

[42] G. J. Walker, "Antibiotics for syphilis diagnosed during pregnancy," Cochrane Database of Systematic Reviews, no. 3, Article ID CD001143, 2001.

[43] M. L. Murray, "Jarisch-Herxheimer reaction," Journal of Obstetric, Gynecologic, and Neonatal Nursing, vol. 25, no. 9, p. 731, 1996.

[44] Y. P. Singh and G. Jalpota, "Jarisch-Herxheimer reaction in early syphilis," Indian Journal of Dermatology, Venereology and Leprology, vol. 61, no. 6, article 386, 1995.

[45] V. R. Klein, S. M. Cox, M. D. Mitchell, and G. D. Wendel Jr., "The Jarisch-Herxheimer reaction complicating syphilotherapy in pregnancy," Obstetrics and Gynecology, vol. 75, no. 3 I, pp. 375-380, 1990.

[46] T. D. Myles, G. Elam, E. Park-Hwang, and T. Nguyen, “The Jarisch-Herxheimer reaction and fetal monitoring changes in pregnant women treated for syphilis," Obstetrics and Gynecology, vol. 92, no. 5, pp. 859-864, 1998.

[47] American Academy of Pediatrics and American College of Obstetricians Gynecologists, "Antepartum care," in Guidelines for Perinatal Care, pp. 87-137, American Academy of Pediatrics, Elk Grove Village, Ill, USA, 6th edition, 2007.

[48] "Istituto Superiore di Sanità," Linee Guida per la gravidanza fisiologica, http://www.snlg-iss.it/cms/files/LG_Gravidanza .pdf, 2011. 


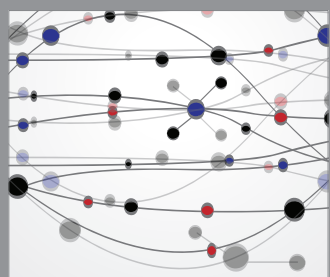

The Scientific World Journal
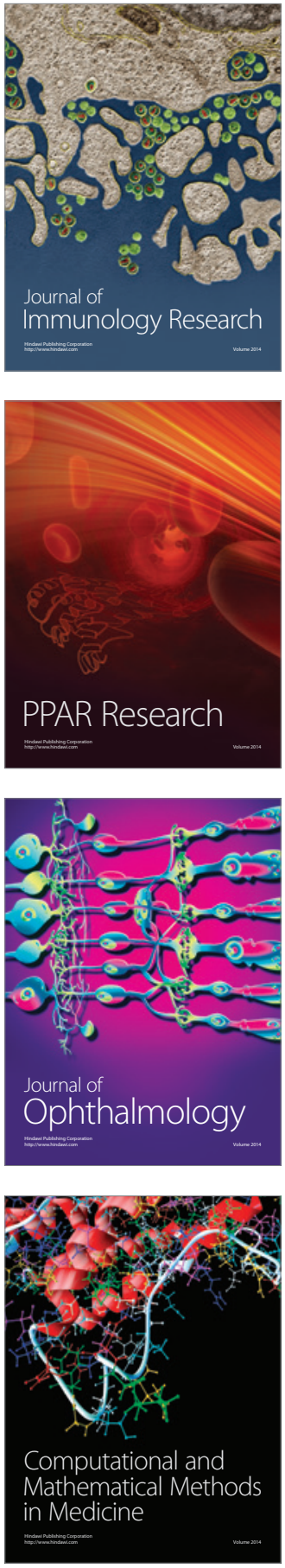

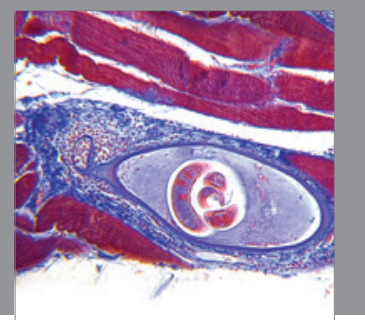

Gastroenterology

Research and Practice
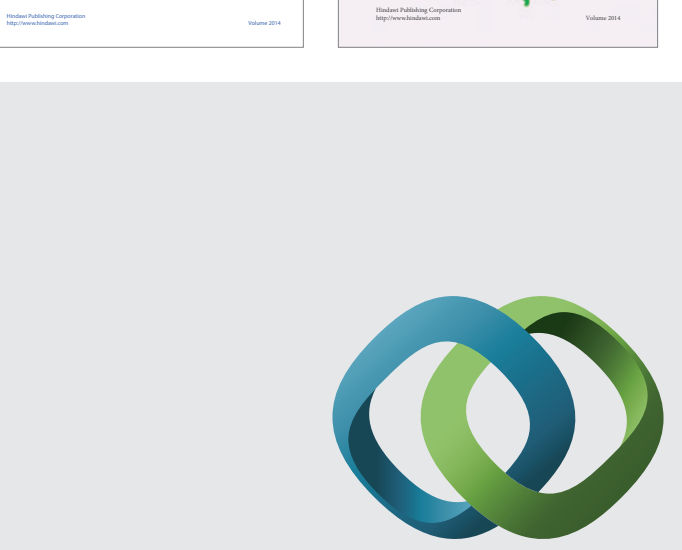

\section{Hindawi}

Submit your manuscripts at

http://www.hindawi.com
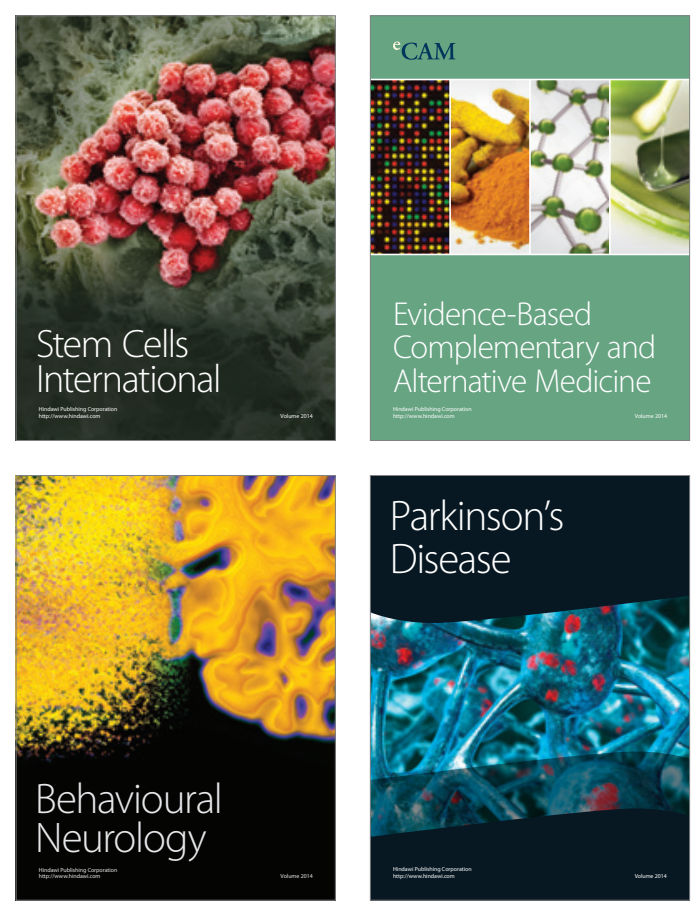

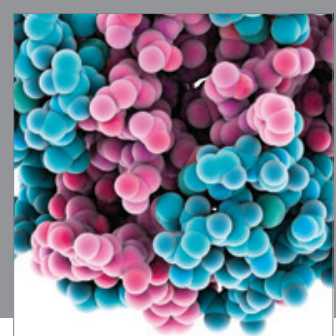

Journal of
Diabetes Research

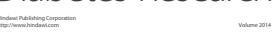

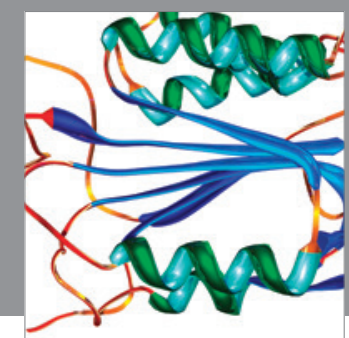

Disease Markers
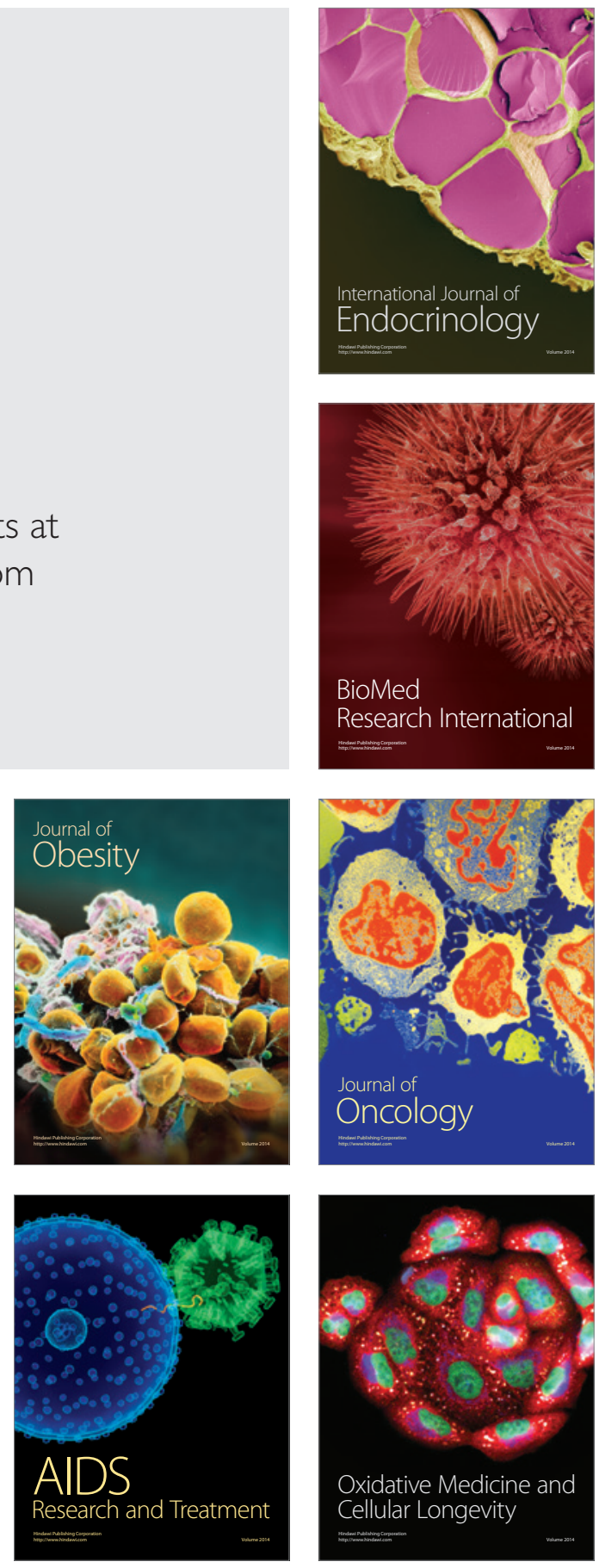